\title{
El uso de la silla maya durante el periodo expulsivo del parto y su relación con la menor incidencia de episiotomía o desgarros perineales ${ }^{1}$
}

\author{
Institución: Universidad de Costa Rica \\ Programa CIEBE-CR
}

\section{COMO CITAR}

\section{RESUMEN}

El objetivo de este trabajo es presentar los resultados de una pregunta clínica acerca del uso de la silla maya o silla obstétrica durante el periodo expulsivo en el parto y su relación con la menor incidencia de episiotomías y prevención de desgarros perineales. En las culturas tradicionales, las mujeres paren de forma natural en posiciones verticales como arrodilladas, paradas, entre otras, mientras que en las sociedades occidentales, los médicos han influido para que las mujeres tengan su parto en decúbito dorsal, algunas veces con las piernas levantadas con estribos, razón por la que para las mujeres de nuestro medio es difícil adoptar posiciones «alternativas» a las que tradicionalmente han visto como «normales». El uso de la silla maya o silla de parto en los países de América del Sur parece ser una manera efectiva para prevenir desgarros perineales y disminuir la incidencia de episiotomía durante el parto. Para llevar a cabo esta investigación secundaria se utilizó la metodología de práctica clínica basada en la evidencia que consta de cinco pasos. Se elaboró una pregunta en formato PICO (paciente, intervención, comparación y observación); posteriormente, se recabó información en bases de datos como Ebsco Host, Google académico, PubMed, Medline plus, Embase. Se encontraron 30 artículos, se analizaron según los criterios que establece FLC, versión 2.0, de los cuales solamente uno se aproximó a responder a la pregunta clínica. Se expuso los datos en una presentación formal realizada por CIEBE-CR. Se concluye que no existe evidencia suficiente que el uso de la silla maya durante el periodo expulsivo reduzca considerablemente el riesgo de desgarro perianal y la menor incidencia de episiotomía durante el parto.

Palabras clave: desgarro-perineal, episiotomía, expulsivo, posición-parto, silla-maya, silla-obstétrica

\footnotetext{
${ }^{1}$ Fecha de recepción: 15 de enero del 2014

Fecha de aceptación: 3 de marzo del 2014

${ }^{2}$ Enfermera. Magíster en Enfermería ginecológica, obstétrica y perinatal. Profesora de la Escuela de Enfermería de la Universidad de Costa Rica. Correo electrónico: lauralopez07@gmail.com

${ }^{3}$ Enfermera. Magíster en Enfermería ginecológica, obstétrica y perinatal. Profesora de la Escuela de Enfermería de la Universidad de Costa Rica. Correo electrónico: anaibisc4@gmail.com
} 


\section{Using maya chair during the expulsive phase of delivery and its relation to the lower incidence of episiotomy or perineal tears 1}

Institution: University of Costa Rica CIEBE-CR Program

\section{RESUMEN}

The aims of this article is to present the results obtained from the development of a clinical question about using maya obstetric chair during the expulsive phase of delivery and its relation to the lower incidence of episiotomy and perineal tears. In traditional cultures, the women give birth naturally in vertical positions as kneeling, standing, among others. In Western societies, physicians have influenced women will deliver in the supine position, sometimes with legs raised using stirrups. For women of our country is difficult to adopt "alternative" positions that have traditionally been seen as 'normal'. The maya chair is used in the countries of South America, and it appears to be an effective way to prevent perineal tears and the lower incidence of episiotomy during childbirth. To carry out this secondary research methodology for clinical practice based on evidence that consists of five steps was used. A question was developed in PICO format (patient, intervention, comparison and observation), then an information search was performed in different databases like Ebsco Host, Google Scholar, PubMed, Medline plus, Embase. 30 articles were found, were analyzed according to the criteria set FCL version 2.0, of which only one was close to answering the clinical question. Information was socialized in a formal presentation by CIEBE-CR. It is conclude that there is insufficient evidence that the use of maya chair during the expulsive phase of delivery significantly reduces the risk of perianal tear and lower incidence of episiotomy.

Keywords: delivery, episiotomy, expulsion, maya-chair, perineal-tear, position, seat-obstetric

\footnotetext{
${ }^{1}$ Date of receipt: January 15, 2014

Date of acceptance: March 3, 2014

${ }^{2}$ Nurse. Master of Nursing gynecologic, obstetric and perinatal. Professor of the School of Nursing. University of Costa Rica. E mail: lauralopez07@gmail.com

${ }^{3}$ Nurse. Master of Nursing gynecologic, obstetric and perinatal. Professor of the School of Nursing. University of Costa Rica. E mail: anaibisc4@gmail.com
} 


\section{INTRODUCCIÓN}

La reproducción humana implica un conjunto de procesos fisiológicos moldeados por la cultura, en virtud de condiciones políticas, materiales y culturales. En general, se interpreta de manera muy diversa y su significado se asocia a la cosmovisión de cada grupo, particularidad que la define como un proceso biocultural (García, $\underline{2012}$.

En un principio, el embarazo, parto y cuidado del bebé ocurría dentro de los hogares, guiado por un acervo de conocimientos que lo dotaban de significación; con la llegada del paradigma médico este proceso salió del ambiente familiar y pasó a desarrollarse en hospitales, manejado por profesionales en obstetricia y siguiendo un procedimiento médico que proviene de un paradigma específico: el científico-técnico.

Como vivencia particular, la posición que adoptan las mujeres durante el parto en occidente responde a diversos factores, especialmente de tipo cultural y que son influenciadas por las instrucciones médicas, motivo por el que resulta difícil determinar cuál sería la conducta instintiva de las mujeres que habitan en países desarrollados. No obstante, en mujeres que viven en zonas menos desarrolladas y que no están tan influidas por la «tecnología» en el parto, se puede observar que adoptan posiciones verticales, como por ejemplo de pie, cuclillas, cuadripedia o de rodillas.

Uno de los instrumentos más antiguos que se ha utilizado, como auxiliar de estas posiciones, es la silla maya. Las sillas específicamente construidas para el parto datan de la época medieval momento en el cual se empleaba una especie de taburete de muy poca altura y con forma de herradura, así lo reseñan Lugones y Ramírez (2011), quienes mencionan cómo en Egipto las mujeres se colocaban de rodillas o sentadas en sillas especiales durante el parto. También se refieren a la época de Hipócrates de Grecia, cuando existían sillas de parto con asiento abierto y respaldo inclinado e identifican el uso de un sillón obstétrico en el Imperio Romano para que la mujer se sentara durante el expulsivo. Además, en los siglos XIII, XIV y XV, en Europa, hay referencias bibliográficas que señalan las sillas de parto con las cuales las mujeres adquirían la posición de cuclillas en lugar de sentarse sobre sí, a lo que aparte hay que sumar que brinda más libertad a la pelvis, así como mayor comodidad.

Una revisión de Cochrane realizada por Gupta, J.K. y Hofmeyr, G. (2008) acerca de la posición de la mujer durante el periodo expulsivo concluye que la posición vertical genera numerosos beneficios potenciales , aunque con la posibilidad de la pérdida sanguínea sea mayor de $500 \mathrm{ml}$. A pesar de los datos, consideran relevante que las mujeres sean quienes escojan la posición más cómoda para ellas, considerando beneficios y riesgos de cada posición; por el momento, hasta que los datos no sean más precisos, queda en manos de cada madre la tarea de informarse acerca del tema.

Tal como en la investigación mencionada, en otras se discute respecto de los beneficios o riesgos de las posiciones de las mujeres durante el periodo expulsivo del parto: según se colige, las posiciones verticales tienen numerosas ventajas al contrastarlas con la posición de litotomía.

Muchos estudios mencionan el uso de la silla obstétrica para ayudar a la mujer a mantener la posición vertical; por ende, se podría inferir que el descanso de los músculos de las piernas apoyadas sobre las sillas de 


\section{Revista Electrónica Enfermería Actual en costa Rica}

parto, más las ventajas de una posición vertical, influyen directamente sobre las estructuras del piso pélvico y los tejidos perineales.

El objetivo de esta revisión fue establecer si el uso de la silla maya durante el periodo expulsivo en el parto disminuye la incidencia de desgarros perineales y episiotomías.

\section{MATERIALES Y MÉTODOS}

Para elaborar esta investigación, se empleó la metodología aplicada en la práctica clínica de enfermería basada en la evidencia la cual, según Coello (2004) citado por Rojas, Cubero y Rodríguez (2013) se define como la "aplicación consciente, explícita y juiciosa de la mejor evidencia científica disponible relativa al conocimiento enfermero para tomar decisiones sobre el cuidados de los pacientes, teniendo en cuenta sus preferencias y valores e incorporando la pericia profesional en esta toma de decisiones" (p. 7).

Este proceso se inicia con la elaboración de la pregunta PICO (persona, intervención, comparación y resultado.), por ende, se procedió a redactar la siguiente pregunta clínica: En las mujeres en periodo expulsivo, ¿el uso de la silla maya comparado con el uso de la cama de parto tradicional disminuye los desgarros perineales o la realización de episiotomías?

Una vez formulada la pregunta clínica, el segundo paso consistió en buscar la información científica disponible, para lo cual se desarrolló una revisión de documentos relacionados con el tema que, según los buscadores universales, incluyeran las palabras claves silla maya, silla de parto, episiotomía, desgarros, cama de parto, tanto en inglés como en español. Para la búsqueda se llevó a cabo en las bases de datos Ebsco Host, Google académico, PubMed, Medline plus, Embase de los que se recuperó 30 documentos. En la tercera etapa se efectuó el análisis crítico de la información, actividad que según Abad (2003), citado por Rojas, Cubero y Rodríguez $\underline{(2013)}$, consiste en

el proceso de evaluar, interpretar, la evidencia aportada por la bibliografía científica, considerando sistemáticamente los resultados que presentan, su validez y su relevancia para el propio trabajo..., a través de la lectura crítica se verifican la validez y aplicabilidad de los resultados publicados con el fin de poder incorporarlos al cuidado de nuestros pacientes(p. 33).

Para el análisis de datos se empleó el programa Fichas de Lectura Crítica (FLC), versión 2.0.; el siguiente paso fue responder a la pregunta de investigación planteada.

El cuarto y quinto paso del proceso corresponden a la implementación de los resultados y su evaluación, por lo que se expuso el trabajo en una presentación formal realizada por CIEBE-CR, aunque resta presentarlos en los centros de salud.

\section{Consideraciones éticas}

La revisión consideró los principios éticos de beneficencia, no maleficencia, justicia y autonomía. Además se respetó los derechos de autor para el manejo de los datos. 


\section{RESULTADOS}

Como resultado de la búsqueda se encontró 30 artículos de los cuales la gran mayoría se refieren a temas relacionados con la pregunta clínica, aunque no de forma específica, por ejemplo tratan de la posición de la mujer durante el periodo expulsivo y los beneficios que obtienen cuando se colocan en posiciones verticales. Entre otros temas aparece el manejo del dolor y la comodidad y algunos aspectos para mejorar las prácticas hospitalarias y la atención del parto humanizado y natural; es decir, con menos intervenciones médicas. Se analizó solamente un documento que se refería directamente al tema en estudio, escrito por Thies-Lagergren, Kvist, Christensson, y Hildingsson (2011), titulado "No reduction in instrumental vaginal births and no increased risk for adverse perineal outcome in nulliparous women giving birth on a birth seat: results of a Swedish randomized controlled trial".

La investigación se desarrolló en diferentes instituciones de Suecia, desde noviembre del año 2006 a julio de 2009; participaron 1002 mujeres y el objetivo consistió en determinar si el uso de un asiento obstétrico o silla maya, durante la segunda etapa del trabajo de parto, para las mujeres nulíparas sanas, disminuía el número de partos asistidos instrumentalmente y si compensaba cualquier aumento de trauma y edema perineal, así como pérdida de sangre, mas se concluye que la silla de parto no reduce el número de partos instrumentados. Por otra parte, se observó un aumento en la pérdida sanguínea de 500 a 1000 cc, sin sobrepasar este límite, en las mujeres que tuvieron su parto en una silla obstétrica. También enfatizan que el parto en una silla obstétrica no tiene consecuencias adversas a nivel perineal y parece ser protector en cuanto a la episiotomía. Según el programa FLC 2.0 , este artículo se clasifica como de alta calidad.

\section{DISCUSIÓN}

En el mundo, se han aunado grandes esfuerzos por aumentar la cantidad de nacimientos por vía vaginal y disminuir la incidencia de cesáreas, para lograrlo se han promovido prácticas como la libertad de movimiento y posiciones durante la labor de parto, el acompañamiento, la acuaterapia y muchas otras, cuyo propósito final es que las madres durante la labor y el periodo expulsivo se sientan seguras y cómodas, aparte de que propicia que la hormona oxitocina, (generadora de contracciones), no sea antagonizada por la adrenalina, producida por el miedo y la inseguridad.

Una práctica que ha demostrado ser beneficiosa para las parturientas son los cambios de posición durante su periodo de dilatación, de tal manera que la madre pueda escoger la postura con la cual se sienta más cómoda durante su periodo expulsivo; una de las posiciones que se promueve es la vertical y sus diversas modalidades: sentada, semisentada o de cuclillas.

En países como Perú, es una práctica que apoya el sistema de salud, en donde la atención del parto en posición vertical se realiza formalmente desde comienzos del decenio de 1990, aunque ya mucho antes se practicaba en los nacimientos domiciliarios. En el año 2005, el Ministerio de Salud de ese país publicó la norma técnica que establece el marco procedimental para la atención del parto vertical (Nureña, 2009); al respecto, cabe mencionar que Costa Rica no se ha quedado atrás: en el año 2009, se publicó la Guía de Atención Integral a las mujeres, niños y niñas, en el periodo prenatal, parto y posparto, en la cual se enfatiza la comodidad de la madre durante el periodo expulsivo. Acerca del tema se afirma que 


\section{Revista Electrónica Enfermería Actual en costa Rica}

Animar a la embarazada para que se sitúe en la posición más cómoda y fisiológica. Respetar la posición que la mujer elija para el periodo expulsivo. Se debe evitar periodos prolongados en posición supina, ya que se ha demostrado que produce mayor dolor, incomodidad, favorece el trauma perineal y vaginal y disminuye la oxigenación fetal (CCSS, 2009, p. 81).

Son muchas las investigaciones que demuestran los beneficios para las madres y sus bebés en posiciones verticales; por ejemplo, Sven, Rake, y Treiber (2001) afirman que los cambios en las posiciones del parto, aumentan las dimensiones pélvicas, lo cual sugiere beneficios obstétricos, específicamente en el corte sagital y diámetro biespinoso que aumentan su calibre de manera significativa en posición de cuclillas.

En cuanto a la posición vertical, Bodner, Bodner, Kimberger, Lozanov, Husslein y Mayerhofer (2003) afirman que hay estudios en donde se demuestra que las mujeres que escogen posiciones alternativas, se sienten más confortables durante la labor y el parto y experimentan menos dolor que las que tienen su parto en la posición supina. Tal información es apoyada por una revisión sistemática de la Cochrane Library acerca de las posiciones distintas de la horizontal en la cual explica que en las otras posiciones disminuye la duración del periodo expulsivo, existe una menor posibilidad de tener un parto instrumental y de tener frecuencias cardíacas fetales anómalas y disminuye el riesgo de una episiotomía; no obstante, hay inconvenientes como mayor posibilidad de desgarro perineal de segundo grado y de sangrados mayores a 500 mililitros:

En algunos resultados hay consistencia en la dirección del efecto, pero diferencias en su estimación, como por ejemplo: la duración del periodo expulsivo (reducciones medias de 4 y 24 minutos), riesgo de episiotomía (riesgos relativos -RR- entre 0,58 y 0,84 ) y de sangrados mayores de $500 \mathrm{ml}$ (RR de 1,68 y 1,92). Respecto de otros resultados no hay consistencia en el hallazgo de diferencias estadísticamente significativas (Gupta, 2012, p.2)

A pesar de los beneficios que se observan en las madres que escogen estas posiciones, especialmente cuclillas o semisentada, según sea la duración del periodo expulsivo esta postura es difícil de mantener durante largos periodos, razón por la que algunas personas recomiendan el uso de la silla de parto o silla maya que brinda soporte a las mujeres en posición vertical. Al respecto, Reid ( 1998) menciona:

Un descubrimiento significante y reciente ha sido el concepto de la habilidad del canal de parto de alterar su forma y tamaño durante la labor, especialmente en la posición de cuclillas...la posición sentada ha sido recientemente retomada con el uso de la silla de parto...lo que permite las ventajas de la posición vertical con buen acceso al perineo por parte de la persona que asiste (p.2).

Las referencias en relación con el uso de la silla maya se encuentran desde hace mucho tiempo en investigaciones históricas que mencionan las prácticas de las mujeres durante el parto: Torres (2003) efectúa una recopilación cultural del parto en el cual es evidente la escogencia por posiciones verticales en el momento del expulsivo y el uso de la silla de parto o silla obstétrica en muchas regiones.

De igual forma lo manifiestan Benito y Rocha (2005) cuando dicen que 
las escasas referencias que a través de la antropología, u otras fuentes se han podido recoger, colocan a la mujer en posición vertical a la hora de parir. En Egipto parece ser que se paría de rodillas o con las mujeres sentadas en sillas especiales. En la época de Hipócrates de Grecia existían sillas de parto con asiento abierto y respaldo inclinado. En el Imperio Romano la mujer daba a luz sentada en un sillón obstétrico. Cuando Cristóbal Colón llegó a América, tuvo ocasión de entrar en contacto con las culturas precolombinas, donde la posición vertical seguía siendo la dominante, bien fuera en cuclillas, arrodillada o sentada. En los siglos XIII, XIV y XV en Europa no hay grandes cambios, y las referencias bibliográficas hablan de sillas de partos ( $\mathrm{p}$. 4).

Queda claro que el uso de las posiciones verticales ha sido un asunto que se practica desde hace mucho tiempo y sus beneficios están todavía siendo estudiados; en cuanto a la silla maya, su mención es poca y su popularidad en el continente americano data no hace mucho tiempo.

Uno de los primeros estudios publicados se desarrolló en el hospital Coombe Lying-in en Dublin desde marzo de 1984 a junio de 1985. El objetivo general era determinar si las nulíparas conducidas en una silla obstétrica tenían menor incidencia de parto instrumentado que aquellas que utilizan una cama convencional; participaron 1250 mujeres nulíparas, gestantes con un único feto vivo, en posición cefálica, sin anestesia epidural y que lograron $10 \mathrm{~cm}$ de dilatación: la principal conclusión es que el parto en una silla maya no ofrece ninguna ventaja obvia en comparación con las mujeres que tienen su parto en una cama convencional.

De igual forma se pueden encontrar otros estudios que concluyen resultados similares en cuanto a la práctica de posiciones verticales, mas nada mencionan en específico de la silla maya.

En el caso del único artículo que aborda la temática, la investigación se desarrolló en Suecia en el año 2011 y se evidencia el uso de la silla maya; no obstante, en cuanto a la incidencia de episiotomía o desgarros no presenta mayores conclusiones, tan solo menciona que el parto en una silla obstétrica no genera consecuencias adversas a nivel perineal y parece ser protector en relación con la episiotomía.

A pesar de la poca contundencia en los resultados de estudios similares, se motiva a las madres y a las instituciones a adoptar la posición vertical durante el parto y, en algunos casos, se recomienda la silla obstétrica, aunque se puede inferir que su uso puede ser un obstáculo respecto de brindar apoyo físico, debido a que es dificultoso para una madre estar en esa misma posición durante largo tiempo, aun cuando sirve para aliviarle el dolor.

Durante el desarrollo de esta investigación se hallaron algunas publicaciones que profundizan la anatomía de las estructuras pelvianas y sus cambios en las diferentes posiciones del parto, mas no se reconocen estudios que analicen dichos cambios relacionados directamente con el uso de la silla obstétrica.

Tampoco los estudios se refieren a los numerosos tipos y diseños de sillas obstétricas que se pueden encontrar en el mercado y que se están utilizando en partos domiciliares y hospitalarios. 


\section{Revista Electrónica Enfermería Actual en costa Rica}

\section{CONCLUSIONES}

Durante el desarrollo de esta investigación no se respondió a la pregunta PICO, aunque de las investigaciones analizadas se pueden inferir dos conclusiones principales:

- No existe evidencia que sustente que el uso de la silla maya disminuya significativamente los desgarros perineales o el procedimiento de episiotomía.

- Existen diferencias en la duración del expulsivo, pérdida sanguínea, presencia de desgarros y utilización de la episiotomía en los partos en posición vertical respecto de los realizados en posición horizontal.

Por lo tanto, al no encontrar evidencia suficiente, es imperante desarrollar una investigación primaria acerca del uso de la silla maya durante el periodo expulsivo del parto.

\section{REFERENCIAS BIBLIOGRÁFICAS}

Benito E., Rocha M. (2005). Posiciones maternas durante el parto. Alternativas a la posición ginecológica. BIOCIENCIAS. Facultad de Ciencias de la Salud. Universidad Alfonso X el Sabio. 28691, Villanueva de la Cañada (Madrid). ISSN: 1696-8077

Bodner, B., Bodner, K., Kimberger, O. et. al. (2003) Women's position during labour: influence on maternal and neonatal outcome. Wien Klin Wochenschr 115(19-20) 720-723.

Caja Costarricense de Seguro Social. (2009). Guía de atención integral a las mujeres, niños y niñas, en el periodo prenatal, parto y posparto.San José: CCSS.

Crowley, P., Elbourne, D., Ashurst, H. (1991). Delivery in an obstetric birth chair: a randomized controlled trial. British Journal of Obstetrics and Gynecology. July 1991, 98, 667- 674.

García, I., Moncayo, S, Sánchez, B. (2012). El parto en México, reflexiones para su atención integral. Ide@s CONSYTEG, 2012.7 (84) 811-844.

García, D. (2012). Representaciones y prácticas sobre el nacimiento: un análisis desde la perspectiva antropológica. La Habana: Editorial Universitaria.

Gupta, J.K., Hofmeyr, G. (2008). Posición de la mujer durante el período expulsivo del trabajo de parto. (Revisión Cochrane traducida). Cochrane Plus, 2008, 4. Oxford: Update Software Ltd. Disponible en: http://www.updatesoftware.com. (Traducida de The Cochrane Library, 2008, Issue 3. Chichester, UK: John Wiley \& Sons, Ltd.).

Hodnett, E., Downe, S., Walsh, D. (2012) Alternative versus conventional institutional settings for birth. Cochrane Database of Systematic Reviews 2012, Issue 8. Art. No.: CD000012. DOI: 10.1002/14651858.CD000012.pub4 
Lugones, M., Ramírez, M. (2011). El parto en diferentes posiciones a través de la ciencia, la historia y la cultura. Policlínico Universitario "26 de julio". La habana. Recuperado de http://bvs.sld.cu/revistas/gin/vol38___ 12/gin15112.htm

Martina, A., Fernández V., Escate L., Sánchez A. et. Al. Parto posición sentada en la Oroya. (1994). Acta Andina 1994, 3 (2), 151-154.

Nureña, C. (2009). Incorporación del enfoque intercultural en el sistema de salud peruano: la atención del parto vertical. Rev. Panam. Salud Pública. 26(4), 368-76.

Reid, J., Harris, N. (1998). Alternative birth positions. Can Fam Physician 1988; 34:1993-1998.

Rojas, L., Cubero, C. y Rodríguez, A. (2013) La investigación en enfermería desde la perspectiva de la evidencia científica: un camino para el mejoramiento de la práctica clínica segura. San José: CIEBE-CR.

Salas, E. (2012). Perspectiva crítica de los antecedentes históricos de la episiotomía. Rev.Enfermería Actual en Costa Rica, 23, 1-7. Recuperado de: http://www. revenf.ucr.ac.cr/episiotomia.pdf

Sven M., Rake, A., Treiber, K., (2002). Obstetric Pelvimetry: Effect of Birthing Position on Pelvic Bony Dimensions. American Roentgen Ray Society. 179.

Thies, L., Kvist, L., Christensson, K. y et. al. (2011). No reduction in instrumental vaginal births and no increased risk for adverse perineal outcome in nulliparous women giving birth on a birth seat: results of a Swedish randomized controlled trial. BMC Pregnancy and Childbirth 11(22) http://www.biomedcentral.com/1471$\underline{2393 / 11 / 22}$

Torres, L. (2003) Antropología y salud : elementos de la tradición sociocultural relacionados con el embarazo, parto y cuidado del recién nacido en mujeres del Programa de Parto Natural, VIII región. Tesis para optar el grado de Licenciatura. Recuperado de http://www.tesis.uchile.cl/tesis/uchile/2003/torres_1/html/index-frames.html 